\title{
Circular arc detection based on Hough transform
}

\author{
Soo-Chang Pei * , Ji-Hwei Horng \\ Department of Electrical Engineering, National Taiwan University, Taipei, Taiwan, ROC \\ Received 10 August 1994; revised 10 January 1995
}

\begin{abstract}
A new method is proposed to detect circular arcs. The detection of a circular arc includes the determination of its center, radius, length, and midpoint. The Hough transform is applied to detect circular arcs by using center and radius as parameters. A peak value in the parameter space of the Hough transform indicates the existence of a circular arc. The center and radius of the circular arc are given by the coordinates of the peak. The arc points are separated from the remaining edge points of the image. Then, the near-peak Hough transform data of the arc points is analyzed to determine the arc length and midpoint.
\end{abstract}

Keywords: Circular arc; Hough transform; Covariance matrix

\section{Introduction}

Circular arcs are important features on the image of industrial parts or tools. Many approaches have been proposed to estimate the arc parameters. The methods proposed in (Landau, 1987) and (Thomas and Chan, 1989) estimate the center and radius of a given set of points by minimizing various error functions. The length and midpoint of the arc (see Fig. 1) are not determined by these methods. Besides, in practical applications, since the arc points are part of the edge points of an image, some preprocess should be presented to separate the set of arc points from the remaining portion in order that these methods are workable. The algorithm proposed in (Saund, 1993) can be applied to identify salient circular arcs, but its input should be a linked list of edge points. The linked list of edge points may be

\footnotetext{
* Corresponding author.
}

hard to be obtained in a complex scene. For example, if the scene contains crossing contours or contours with two or more pixel width, the edge linking process will be difficult and complex.

The Hough transform can be used to detect circular arcs by choosing center and radius as parameters (Brown, 1983; Casasent and Krishnapuram, 1987; Sklansky, 1978). The location of a peak value in the parameter space indicates the contour circle where an arc lies upon. That is, the center and radius of the arc are acquired. The height of the peak indicates the arc length, but this is not valid under noisy environ-

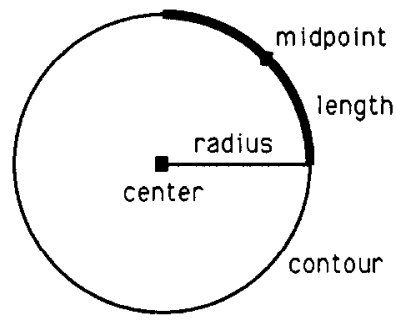

Fig. 1. The arc detection problem. 
ment. Furthermore, the arc midpoint (which portion of the contour where the arc lies upon) cannot be determined from the location and the height of the peak.

The arc length and midpoint can be computed from the near-peak Hough transform data. Similar researches on extracting straight line position (midpoint of a line segment) and length have been proposed in (Richards and Casasent, 1991; Akhtar and Atiquzzaman, 1992; Nagao and Nakajima, 1987). But, these approaches cannot be extended directly to detect circular arcs, especially when the circular arc is longer than a semicircle.

In this paper, we analyze the properties of the covariance matrix and the centroid of the near-peak Hough transform data. The arc parameters are estimated by using the Hough transform and these properties. As in most of the applications of the Hough transform, there is no particular restriction on the input image of edge points. In Section 2, we review the technique of detecting circles by using the Hough transform. The definition of covariance matrix and a related theorem are given in Section 3. Some properties of the near-peak Hough transform data and the method proposed by us for determining the arc length and midpoint are given in Section 4. Section 5 provides simulation results. Section 6 presents the conclusion of this paper.

\section{Detecting circles by using Hough transform}

The Hough transform is a mapping from the image plane onto the parameter space. The parameter space is quantized into an accumulator array, and each accumulator stands for the curve specified by the coordinates of the accumulator. For each edge point on the image plane, the curves passing through the point are computed, and the accumulators corresponding to these curves are incremented by 1 . After the transform, the accumulator with a peak value indicates the existence of a curve, which is specified by the coordinates of the accumulator, on the image plane.

The circle with center $\left(x_{0}, y_{0}\right)$ and radius $r_{0}$ is specified by the parameters $\left(x_{0}, y_{0}, r_{0}\right)$ in the equation

$$
\left(x-x_{0}\right)^{2}+\left(y-y_{0}\right)^{2}=r_{0}^{2} \text {. }
$$

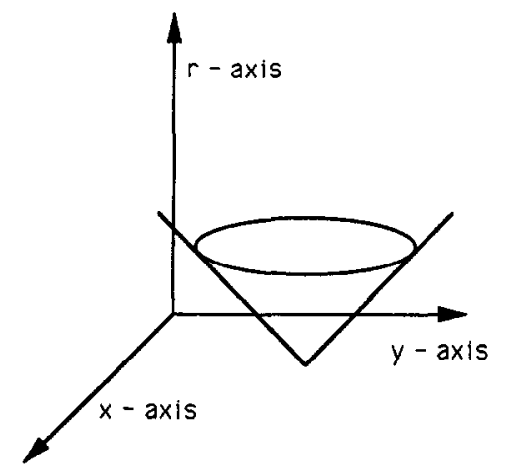

Fig. 2. Circular Hough transform voted by an edge point.

Choosing $\left(x_{0}, y_{0}, r_{0}\right)$ as the parameters of the Hough transform, the locus of the accumulators, in the parameter space, incremented by an edge point on the image plane is a right circular cone (see Fig. 2). If there is a circle on the image plane, all the right circular cones incremented by the edge points of the circle will intersect at a common accumulator in the parameter space. The coordinates of this common accumulator are the parameters of the equation for the circle on the image plane.

The Hough transform is capable of detecting partial shapes. Treating a circular arc as a partial shape of a circle, the Hough transform for detecting circles can be applied to detect circular arcs. A peak value in the accumulator array indicates the existence of a circular arc. The center and radius of the detected arc are given by the coordinates of the peak.

\section{The covariance matrix}

Let $S$ be a set of points, the mass of $S$ at the point $(x, y)$ is given by $f(x, y)$.

Definition 1 (Moment). The $(p, q)$ moment of $S$ is defined (Rosenfeld and Kak, 1982) as

$m_{p q}=\sum_{x} \sum_{y} x^{p} y^{q} f(x, y)$.

Definition 2 (Central moment). The $(p, q)$ central moment of $S$ is defined as

$$
M_{p q}=\sum_{x} \sum_{y}(x-\bar{x})^{p}(y-\bar{y})^{q} f(x, y),
$$


where $(\bar{x}, \bar{y})$ is the centroid of $S$ and is given by $(\bar{x}, \bar{y})=\left(m_{10} / m_{00}, m_{01} / m_{00}\right)$.

Definition 3 (Covariance matrix). The covariance matrix of $S$ is defined (Tsai and Chou, 1991) as

$\left[\begin{array}{ll}M_{20} & M_{11} \\ M_{11} & M_{02}\end{array}\right]$

Theorem 1. If $S$ is symmetric about the $y$-axis, then the $(1,1)$ central moment of $S$ must be zero.

Proof. See Appendix A.

Corollary 1. The axis of symmetry is an eigenvector of the covariance matrix.

\section{Determining the arc midpoint}

\subsection{Problem formulation}

In this section, we assume that the center $\left(x_{0}, y_{0}\right)$ and radius $r_{0}$ of an arc have been determined by using the Hough transform technique provided in Section 2.

Definition 4 (Near-peak Hough transform data, see Fig. 3). The near-peak Hough transform data is defined as the data stored in those accumulators lying on the accumulator disk

$h\left(x, y, r_{0}\right)$ and $\sqrt{x^{2}+y^{2}} \leqslant \delta$,

where $h\left(x, y, r_{0}\right)$ is a cross-section, at $r=r_{0}$, of the

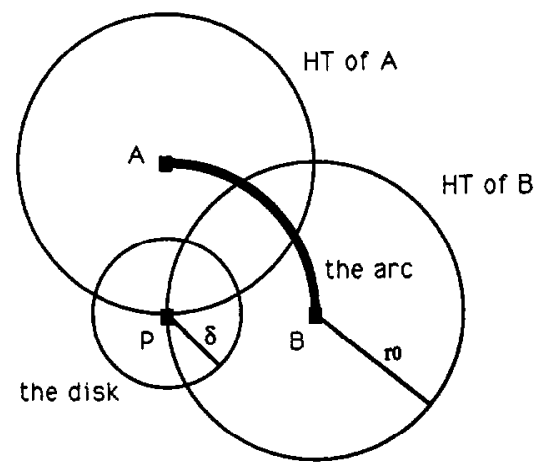

Fig. 3. The near-peak Hough transform data (only votes of $A$ and B are shown).

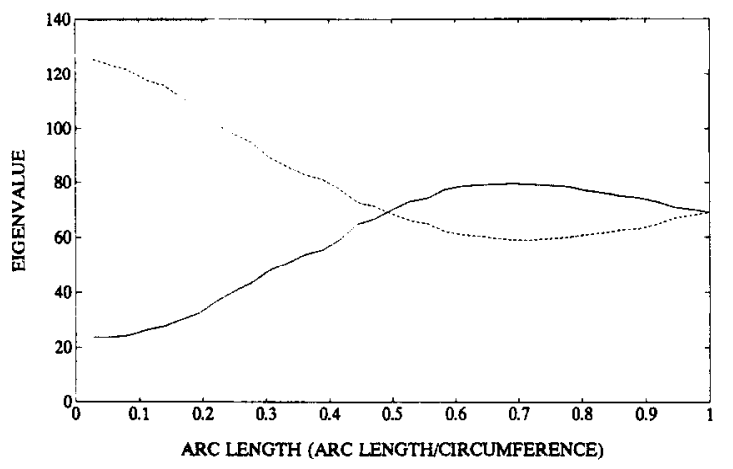

Fig. 4. The eigenvalues versus the arc length.

three-dimensional accumulator array $h(x, y, r)$ produced by the Hough transform, and $\delta$ is the radius of the accumulator disk. The value of $\delta$ is typically chosen equal to $r_{0}$ or some value comparable with $r_{0}$.

In this paper, we focus our efforts on determining the arc midpoint by analyzing the near-peak Hough transform data. In addition, the estimation of the arc length under noisy environment can be improved by using the near-peak Hough transform data.

\subsection{Symmetry and centroid}

Theorem 2. The near-peak Hough transform data of a circular arc is symmetric about the line passing through the center and midpoint of the arc.

Proof. See Appendix B.

Corollary 2. The vector $\left[x_{\mathrm{m}}-x_{0}, y_{\mathrm{m}}-y_{0}\right]$, where $\left(x_{\mathrm{m}}, y_{\mathrm{m}}\right)$ and $\left(x_{0}, y_{0}\right)$ are the midpoint and center of the circular arc respectively, is an eigenvector of the covariance matrix of the near-peak Hough transform data $h\left(x, y, r_{0}\right)$.

Remark. In fact, if the circular arc is shorter than a semicircle, the vector $\left[x_{\mathrm{m}}-x_{0}, y_{\mathrm{m}}-y_{0}\right]$ will be the eigenvector corresponding to the smaller eigenvalue. On the contrary, if it is longer than a semicircle, the vector $\left[x_{\mathrm{m}}-x_{0}, y_{\mathrm{m}}-y_{0}\right]$ will be the eigenvector corresponding to the larger eigenvalue. An experimental result is plotted in Fig. 4. 
Theorem 3. The centroid of the near-peak Hough transform data lies on the line segment connecting the center with the midpoint of the circular arc.

Proof. See Appendix C.

Remark. Although Theorem 3 can be used to determine the arc midpoint uniquely, in practical applications, it is sensitive to noise even if there is quantization noise only. On the other hand, the eigenvectors are more robust under noisy environment, but ambiguity occurs when we try to determine the arc midpoint. Our proposed method takes advantage of both quantities to determine the arc midpoint uniquely.

\subsection{Noise and nearby curves}

Under noisy environment, the peak value of the Hough transform data may spread into its neighbouring accumulators. Thus, the arc length is no longer equal to the peak value. The arc length can be calculated based on the formula derived in Appendix C.

It can be proven that the estimation is robust to noise provided that the distribution of noise is unbiased. That is, if an arc point is displaced, the proba-

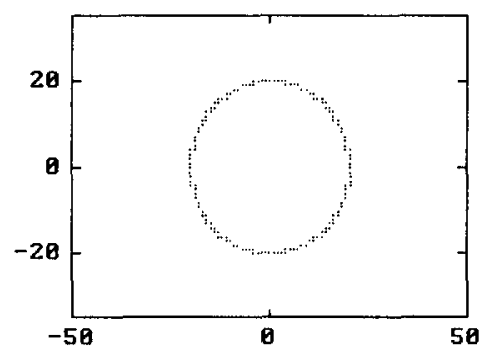

(a)

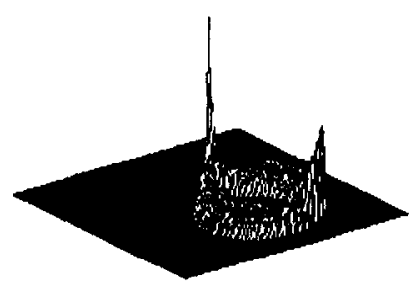

(c) bilities of displacing to its eight nearest neighbours are equally alike; if some arc points are missed, the missing probabilities for all of the arc points are equally alike; and so on.

If the image contains multiple curves including the circular arcs, the curves nearby the detected circular arc will vote on the near-peak region of the arc. The overlapping distributions will result in incorrect estimation of the parameters. A preprocess is required to remove the nearby curves before the near-peak Hough transform is performed, which is included in the following procedure.

\subsection{The detection procedure}

\section{Step 1: Performing the Hough transform}

A circular arc is treated as a partial shape of a circle. The Hough transform is applied to detect the contour where the arc lies upon.

Step 2: Detecting the location of the peak value

The location of a peak value, in the three-dimensional accumulator array of the Hough transform, indicates the center and radius of the detected circular arc. Notice that, if the image contains concentric circular arcs, the accumulator array will have two

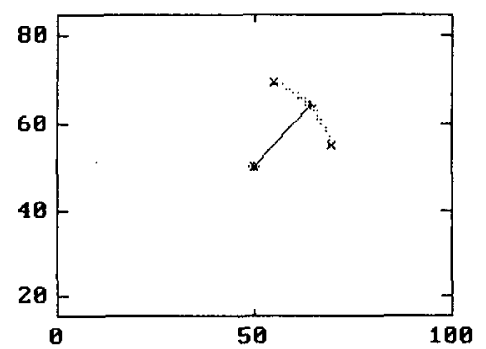

(b)

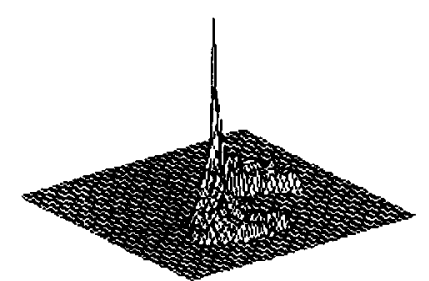

(d)

Fig. 5. (a) A circle. (b) The input arc and the detected result. (c) The Hough transform data. (d) The near-peak Hough transform data. 


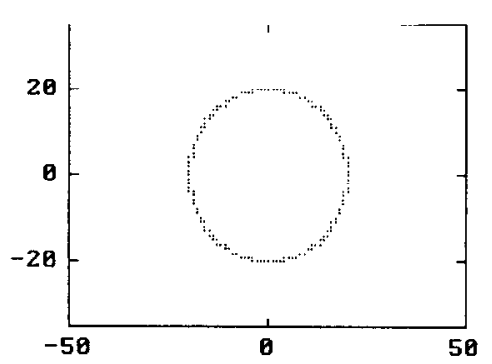

(a)

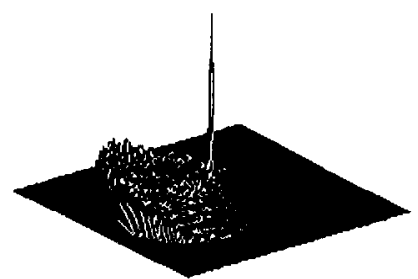

(c)

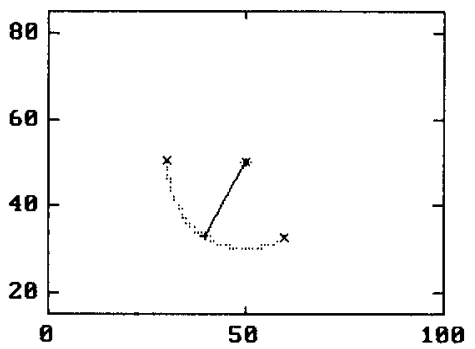

(b)

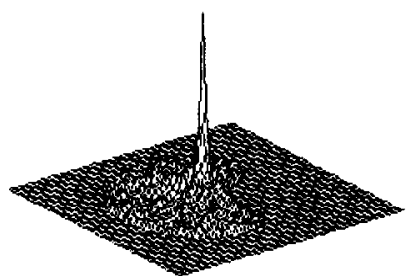

(d)

Fig. 6. (a) A circle. (b) The input arc and the detected result. (c) The Hough transform data. (d) The near-peak Hough transform data.

local peaks at the same coordinates $x$ and $y$ but at different coordinate $r$. That is, concentric arcs can be resolved by the parameter $r$.

Step 3: Removing the nearby curves

The points, on the image, outside the ring containing the contour circle where the detected arc lies upon are removed. The width of the ring is chosen to

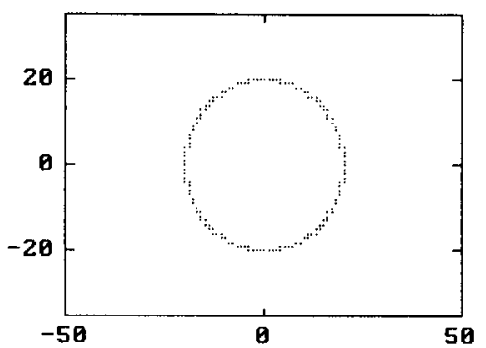

(a)

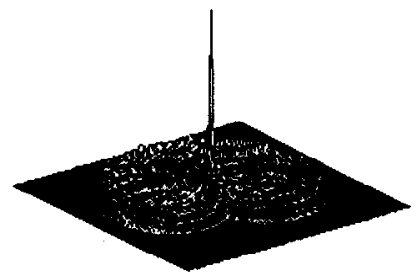

(c) be 5 pixels or so, such that the noise degraded arc is fully contained in the ring belt.

Step 4: Performing the Hough transform of the resulting image

The Hough transform of the resulting image is performed. This time the near-peak Hough transform data is no longer distorted by the nearby curves.

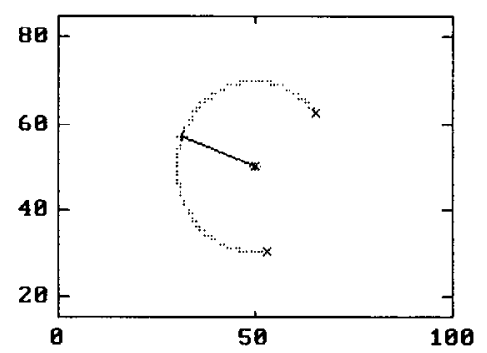

(b)

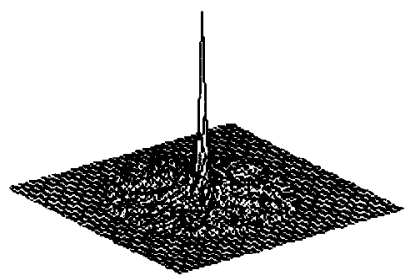

(d)

Fig. 7. (a) A circle. (b) The input arc and the detected result. (c) The Hough transform data. (d) The near-peak Hough transform data. 
Table 1

The estimation of arc midpoint using different methods

\begin{tabular}{llll}
\hline Input arc & Fig. 5(b) & Fig. 6(b) & Fig. 7(b) \\
\hline $\begin{array}{l}\text { Angle of } \\
\text { midpoint } \\
\text { (degree) }\end{array}$ & 45 & -120 & 160 \\
$\begin{array}{l}\text { Estimation } \\
\text { using } \\
\text { centroid }\end{array}$ & 45 & -121.42 & 158.75 \\
$\begin{array}{l}\text { Estimation } \\
\text { using } \\
\text { eigenvector }\end{array}$ & 45 & -120.87 & 159.04 \\
\hline
\end{tabular}

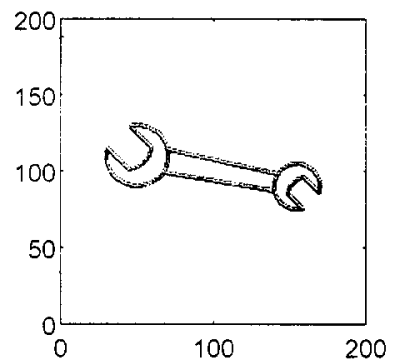

(a)

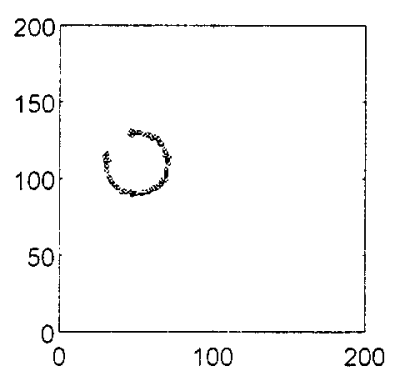

(c)

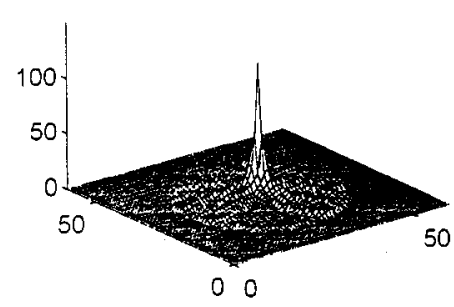

(e)
Step 5: Calculating eigenvectors of the covariance matrix

The covariance matrix of the near-peak Hough transform data is calculated. One of its eigenvectors directs to the midpoint of the detected circular arc. Step 6: Calculating the centroid of the near-peak Hough transform data

In order to solve the ambiguity in determining the arc midpoint and to estimate the arc length, the centroid of the near-peak Hough transform data is calculated.
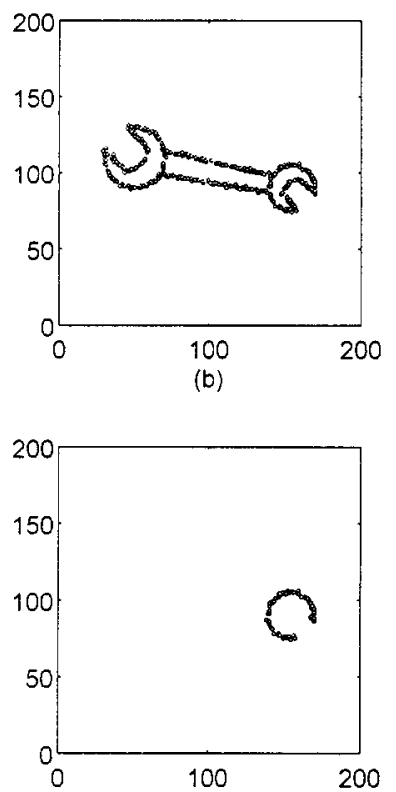

(d)

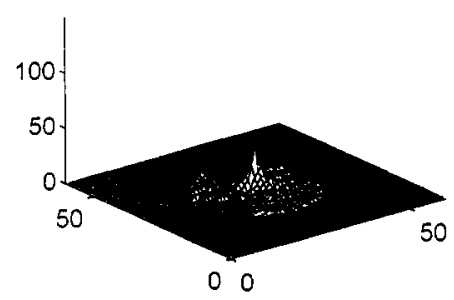

(f)

Fig. 8. (a) The computer synthesized digital image of a spanner. (b) The noise corrupted version of (a). (c), (d) The circular arcs extracted from (b) by using the Hough transform. (e), (f) The near-peak Hough transform data of (c) and (d) respectively. 


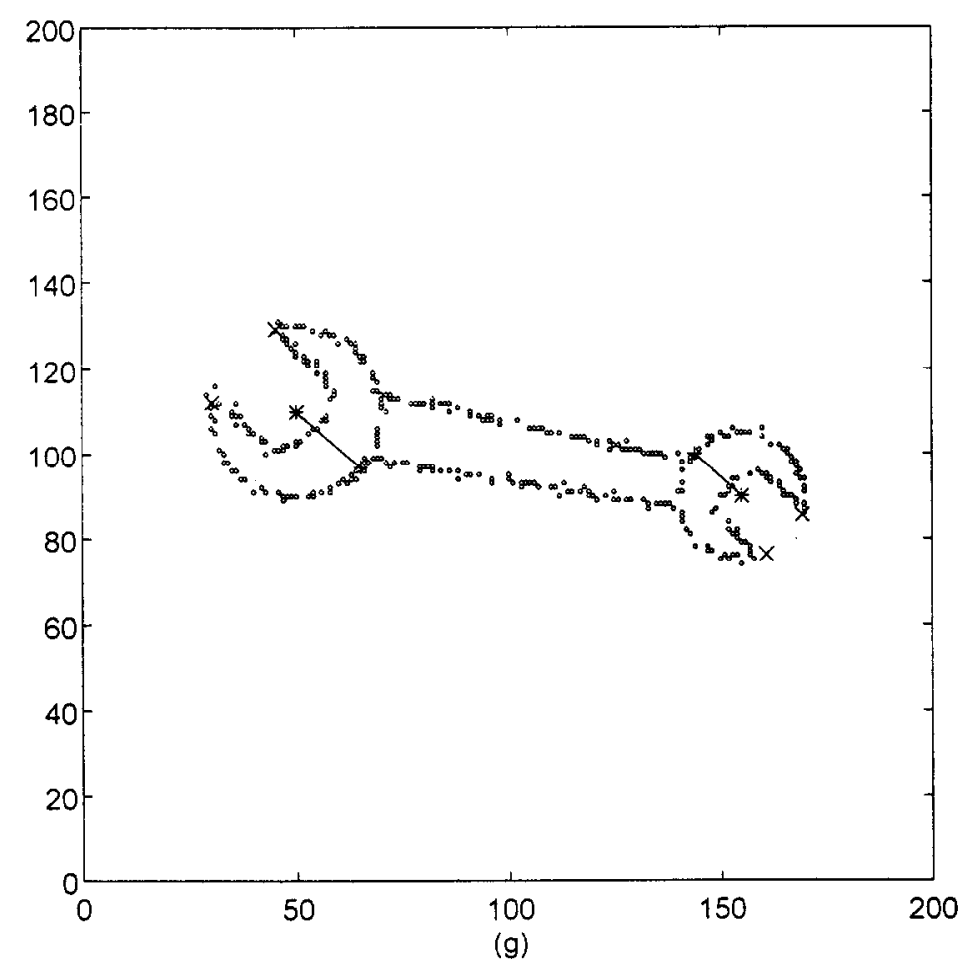

Fig. 8. (g) The estimated parameters: arc centers are marked by $*$, arc midpoints are marked by + , and end points are marked by $\times$.

\section{Step 7: Estimating the arc length}

The arc length is calculated based on the formula derived in Appendix C.

Step 8: Locating the arc midpoint

Let $E_{\lambda 1}$ and $E_{\lambda 2}$ be the two orthonormal eigenvectors of the covariance matrix, the location of the arc midpoint is

$$
\begin{aligned}
M= & T+r_{0} \max \left\{\frac{\overrightarrow{T C}_{A B} \cdot E_{\lambda 1}}{\left|\overrightarrow{T C}_{A B}\right|},-\frac{\overrightarrow{T C}_{A B} \cdot E_{\lambda 1}}{\left|\overrightarrow{T C}_{A B}\right|},\right. \\
& \left.\frac{\overrightarrow{T C}_{A B} \cdot E_{\lambda 2}}{\left|\overrightarrow{T C}_{A B}\right|},-\frac{\overrightarrow{T C}_{A B} \cdot E_{\lambda 2}}{\left|\overrightarrow{T C}_{A B}\right|}\right\},
\end{aligned}
$$

where $T$ and $C_{A B}$ are the location of the peak value and the centroid of the near-peak Hough transform data respectively.

Remark. If the property described in the remark of Corollary 2 is applied to choose a suitable eigenvec- tor $E_{\lambda}$ from $E_{\lambda 1}$ and $E_{\lambda 2}$, then the equation in Step 8 can be simplified into

$$
M=T+r_{0} \max \left\{\frac{\overrightarrow{T C}_{A B} \cdot E_{\lambda}}{\left|\overrightarrow{T C}_{A B}\right|},-\frac{\overrightarrow{T C}_{A B} \cdot E_{\lambda}}{\left|\overrightarrow{T C}_{A B}\right|}\right\}
$$

\section{Simulations}

\subsection{A single circular arc}

The digital image of a circle is shown in Fig. 5(a). The circular arc plotted in Fig. 5(b) is a partial shape of the circle plotted in Fig. 5(a). The Hough transform data of the circular arc at the cross-section $r=r_{0}$ of the accumulator array, where the peak value belongs to, is plotted in Fig. 5(c). The location of the peak value on the accumulator array indicates the center of the circular arc, which is marked by a 
star (*) in Fig. 5(b). The near-peak Hough transform data is shown in Fig. 5(d), where the radius of the disk $\delta$ is chosen equal to the radius of the circle $r_{0}$. Eigenvectors of the covariance matrix and the centroid of the near-peak Hough transform data are then calculated. The arc length and midpoint can be estimated from these data by using the procedure proposed in the previous section. The arc midpoint is marked by a plus $(+)$ in Fig. 5(b), and the two end points of the circular arc are marked by crosses $(X)$. Two additional simulations are shown in Figs. 6 and 7.

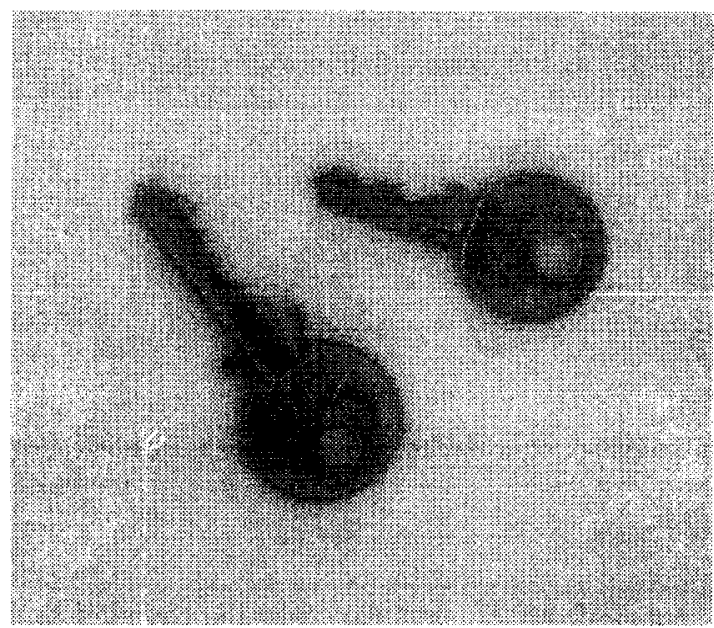

(a)
The accuracy of the angle $\tan ^{-1}\left(\left(y_{\mathrm{m}}-y_{0}\right) /\left(x_{\mathrm{m}}\right.\right.$ $\left.\left.-x_{0}\right)\right)$ estimated from the eigenvector is compared with the estimation from the centroid in Table 1, which confirms the remark of Theorem 3 .

\subsection{Multiple circular arcs in a noisy scene}

The computer-synthesized digital image of a spanner is shown in Fig. 8(a). The noise corrupted version is shown in Fig. 8(b), where $20 \%$ of the edge points in Fig. 8(a) are missed and 30\% of the edge points are displaced to one of its eight nearest neigh-

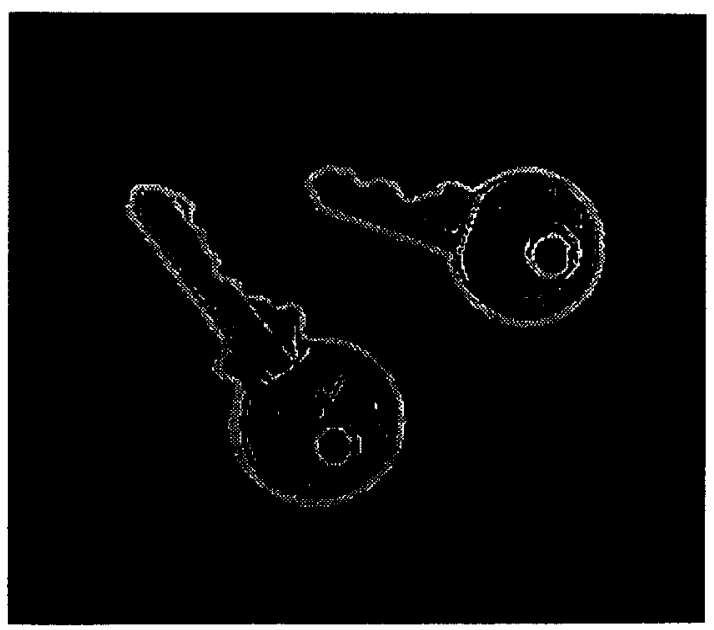

(b)

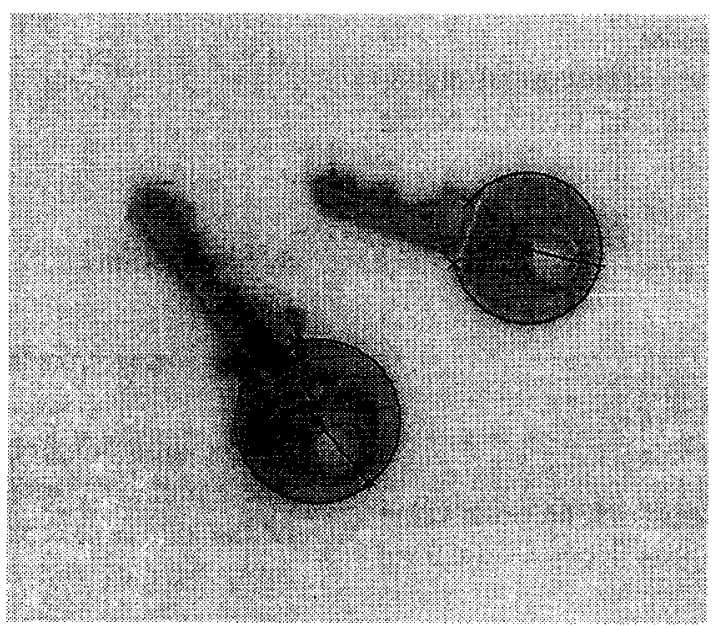

(c)

Fig. 9. (a) The real image of two keys. (b) The edge points detected by Sobel edge detector. (c) The detection results, where the arc centers are marked by stars $(*)$, the arc midpoints are marked by plus symbols $(+)$, and the end points are marked by crosses $(x)$. 
Table 2

The actual values and estimated values of the arc parameters.

\begin{tabular}{lcc}
\hline & Actual value & Estimated value \\
\hline $\begin{array}{l}\text { Length } / 2 r_{0} \\
\text { (degree) }\end{array}$ & 150 & 145 \\
$\begin{array}{l}\text { Angle of } \\
\text { midpoint } \\
\text { (degree) }\end{array}$ & -45 & -41 \\
$\begin{array}{l}\text { Length } / 2 r_{0} \\
\text { (degree) }\end{array}$ & 150 & 155 \\
$\begin{array}{l}\text { Angle of } \\
\text { midpoint } \\
\text { (degree) }\end{array}$ & 135 & 138 \\
\hline
\end{tabular}

bours at random. The circular arcs detected are separated from the remaining edge points and plotted in Figs. 8(c) and 8(d). The locations of the peak values on the accumulator array indicate the centers of the detected circular arcs, which are marked by stars $(*)$ in Fig. $8(\mathrm{~g})$. The near-peak Hough transform data corresponding to the arcs in Figs. 8(c) and 8(d) are shown in Figs. 8(e) and 8(f) respectively, where the radius of the disk $\delta$ is chosen equal to the radius of the circle $r_{0}$. Eigenvectors of the covariance matrices and the centroids of the near-peak Hough transform data are then computed. The arc lengths and midpoints can be estimated from these data by using the procedure proposed in the previous section. The arc midpoints are marked by plus symbols $(+)$ in Fig. $8(\mathrm{~g})$, and the end points of the circular arcs are marked by crosses $(x)$. The values of the arc parameters and the estimated values are tabulated in Table 2.

\subsection{Real image}

A real image, of size $261 \times 300$, containing two keys is shown in Fig. 9(a). The edge points shown in Fig. 9(b) are detected by the Sobel edge detector. The selected threshold is $30 \%$ of the peak gradient value. The detected circular arcs are plotted in Fig. 9(c). In application to real images, missed and doubled segments may occur. To reduce these phenomena, a smaller threshold value is selected and an image ring with narrower width is cut down at Step 3 of the detection procedure. The smaller threshold value permits more points to be selected as edge points, the possibility of missing segments is reduced. The narrower ring width reduces the possibility of containing double segments in a ring.

\section{Conclusion}

The Hough transform is applied to detect the existence of a circular arc and the contour it lies upon. The length of the detected circular arc and the location of the arc on its contour are estimated from the near-peak Hough transform data. We have presented theoretical analysis and proposed a detection procedure to make an accurate estimation of the arc length and midpoint. Simulation results are presented to demonstrate the applicability of the approach.

\section{Appendix}

\section{A. Proof of Theorem 1}

If we substitute $-x$ for $x$ in the definition of $\boldsymbol{M}_{11}$, we obtain

$$
\begin{aligned}
M_{11} & =\sum_{x} \sum_{y}(x-\bar{x})(y-\bar{y}) f(x, y) \\
& =\sum_{x} \sum_{y}(-x-\bar{x})(y-\bar{y}) f(-x, y) .
\end{aligned}
$$

Since $S$ is symmetric about the $y$-axis, $\bar{x}=0$ and $f(-x, y)=f(x, y)$.

Substituting 0 for $\bar{x}$ and $f(x, y)$ for $f(-x, y)$ in the above equation, gives

$$
\begin{aligned}
M_{11} & =\sum_{x} \sum_{y} x(y-\bar{y}) f(x, y) \\
& =\sum_{x} \sum_{y}-x(y-\bar{y}) f(x, y)=-M_{11}=0 .
\end{aligned}
$$

\section{B. Proof of Theorem 2}

The Hough transform data at $r=r_{0}$ can be written as

$$
\begin{aligned}
& h\left(x, y, r_{0}\right) \\
& \quad=\sum_{x^{\prime}} \sum_{y^{\prime}} q\left(x^{\prime}-x, y^{\prime}-y, r_{0}\right) p\left(x^{\prime}, y^{\prime}\right),
\end{aligned}
$$


where

$p(x, y)= \begin{cases}1 & \begin{array}{l}\text { if }(x, y) \text { is an edge point on the } \\ \text { image plane, } \\ 0\end{array} \\ \text { otherwise. }\end{cases}$

$q\left(x, y, r_{0}\right)= \begin{cases}1 & \text { if }(x, y) \text { is a point on the } \\ \text { digital curve } x^{2}+y^{2}=r_{0}^{2} & \begin{array}{l}\text { otherwise. }\end{array}\end{cases}$

Substituting $-x$ for $x$ in the above equation, we obtain

$$
\begin{aligned}
& h\left(-x, y, r_{0}\right) \\
& \quad=\sum_{x^{\prime}} \sum_{y^{\prime}} q\left(x^{\prime}+x, y^{\prime}-y, r_{0}\right) p\left(x^{\prime}, y^{\prime}\right) .
\end{aligned}
$$

Substituting dummy variable $-x^{\prime \prime}$ for $x^{\prime}$,

$$
\begin{aligned}
& h\left(-x, y, r_{0}\right) \\
& \quad=\sum_{x^{\prime \prime}} \sum_{y^{\prime}} q\left(-x^{\prime \prime}+x, y^{\prime}-y, r_{0}\right) p\left(-x^{\prime \prime}, y^{\prime}\right) \\
& =\sum_{x^{\prime \prime}} \sum_{y^{\prime}} q\left(-\left(x^{\prime \prime}-x\right), y^{\prime}-y, r_{0}\right) p\left(-x^{\prime \prime}, y^{\prime}\right) .
\end{aligned}
$$

Assuming the center and midpoint of the arc lie on the $y$-axis without loss of generality, then

$p(-x, y)=p(x, y)$.

The digital curve $x^{2}+y^{2}=r_{0}^{2}$ is symmetric about the $y$-axis, that is

$q\left(-x, y, r_{0}\right)=q\left(x, y, r_{0}\right)$.

Therefore,

$$
\begin{aligned}
& h\left(-x, y, r_{0}\right) \\
& \quad=\sum_{x^{\prime \prime}} \sum_{y^{\prime}} q\left(x^{\prime \prime}-x, y^{\prime}-y, r_{0}\right) p\left(x^{\prime \prime}, y^{\prime}\right) \\
& \quad=h\left(x, y, r_{0}\right) .
\end{aligned}
$$

\section{Proof of Theorem 3}

The Hough transform data, at the cross-section $r=r_{0}$, produced by an edge point $S$ is shown in Fig. 10. Assume that the point $T$ is the location of the peak value, and the disk with center $T$ and radius $\delta$ is the near-peak region. The arc $P Q$ is the part of the near-peak Hough transform data produced by the point $S$ and is symmetric about the line $S T$. The angle $\phi$, which is half of the angle corresponding to

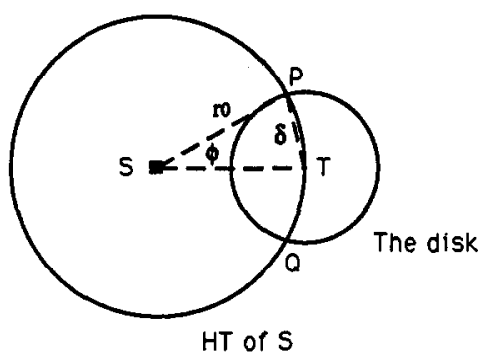

Fig. 10. The circular Hough transform of an edge point.

the votes of $S$ within the disk, can be computed from $r_{0}$ and $\delta$. The equation is given by

$\phi=\cos ^{-1}\left(\frac{2 r_{0}^{2}-\delta^{2}}{2 r_{0}^{2}}\right)$.

The centroid $C_{S}$ of the arc $P Q$ lies on the line segment $S T$ and the length of the line segment $S C_{S}$ can be computed by

$d_{S}=\frac{1}{2 \phi} \int_{-\phi}^{\phi} r_{0} \cos \phi^{\prime} \mathrm{d} \phi^{\prime}=r_{0} \frac{\sin \phi}{\phi}$.

Thus, the centroid of the near-peak Hough transform data produced by any edge point on the circular arc $A B$, shown in Fig. 11, must lie on the arc $C D$, whose radius is $r_{0}-d_{S}$, and all of these centroids have the same weight. Therefore, the centroid $C_{A B}$ of the near-peak Hough transform data produced by the circular arc $A B$ lies on the line segment $T E$, where the point $E$ is the midpoint of the circular arc $A B$. The length of the line segment $T C_{A B}$, which is the distance between the centroid and peak position, is

$d=\left(r_{0}-d_{s}\right) \frac{\sin \psi}{\psi}=r_{0}\left(1-\frac{\sin \phi}{\phi}\right) \frac{\sin \psi}{\psi}$,

where $\psi$ is half of the angle $A T B$.

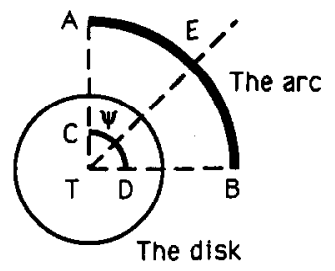

Fig. 11. The centroid of the ncar-peak data. 


\section{References}

Akhtar, M.W. and M. Atiquzzaman (1992). Determination of line length using Hough transform, Electronics Lett. 28 (1), 94-96.

Brown, C.M. (1983). Inherent bias and noise in the Hough transform. IEEE Trans. Pattern Anal. Mach. Intell. 5 (5) 493-505.

Casasent, D. and R. Krishnapuram (1987). Curved object location by Hough transformations and inversions. Pattern Recognition 20 (2), 181-188.

Landau, U.M. (1987). Estimation of circular arc center and its radius. Comput. Vision Graphics Image Process. 38, 317-326.

Nagao, M. and S. Nakajima (1987). On the relation between the Hough transformation and the projection curves of a rectangular window. Pattern Recognition Lett. 6 (3), 185-188.
Richards, J. and D.P. Casasent (1991). Extracting input-line position from transform data. Appl. Opt. 30 (20), 2899-2905.

Rosenfield, A. and A.C. Kak (1982). Digital Picture Processing, Vol. II. Academic Press, New York.

Saund, E. (1993). Identifying salient circular arcs on curves. CVGIP: Image Understanding 58 (3), 327-337.

Sklansky, J. (1978). On the Hough technique for curve detection. IEEE Trans. Comput. 27 (10), 923-926.

Thomas, S.M. and Y.T. Chan (1989). A simple approach for the estimation of circular arc center and its radius. Comput. Graphics Image Process. 45, 362-370.

Tsai, W.-H. and S.-L. Chou (1991). Detection of generalized principal axes in rotationally symmetric shapes. Pattern Recognition 24 (2), 95-104. 